\title{
Correction to: Abstracts
}

\section{9th European Congress of Pathology}

\author{
L. Mikhaleva ${ }^{1} \cdot$ A. Birukov ${ }^{1}$ N. Shakhpazyan ${ }^{2} \cdot$ R. Vandysheva $^{1}$
}

Published online: 16 November 2017

(C) Springer-Verlag GmbH Deutschland 2017

Correction to: Virchows Archiv (2017) 471(Suppl 1):S1-S352 https://doi.org/10.1007/s00428-017-2205-0

\section{PS-13-116}

Early cancer of the stomach: Features of detection of Helicobacter pylori

L. Mikhaleva*, A. Birukov, N. Shakhpazyan, R. Vandysheva *Institute of Human Morphology, Moscow, Russia

Objective: To study the features of the detection of Helicobacter pylori (Hp) in early gastric cancer (EGC) by means of Romanovsky-Giemsa stain (RG), immunohistochemical (IHC) study and real-time polymerase chain reaction (PCR).

Method: The material for the study was fragments of the gastric mucosa with early cancer from 21 patients. 3 methods were used for the detection of Hp: RG staining, IGH study using Cell Marque rabbit polyclonal antibodies to $\mathrm{Hp}$ and real-time PCR using AmpliSens Hp kit.

Results: In $66.5 \%$ of cases Hp were not detected by any of the methods of research, and in 33.5\% - Hp were detected by at least one of the methods. In this case, Hp were detected in $29 \%$ of positive cases by all three methods; also in $29 \%$ of positive cases Hp were diagnosed only by PCR. In the remaining $42 \%$ of cases Hp were detected by two methods of three. The sensitivity of PCR is $86 \%$, RG is $71.5 \%$, in IHC it is $43 \%$. The expressed dissemination of $\mathrm{Hp}(+++)$ was only $14 \%$ of all positive cases, in $86 \%$ cases - weak (+).

Conclusion: Only in $1 / 3$ of patients with EGC Hp was detected, and in $86 \%$ of them - weak dissemination Hp. At the same time, none of the using methods gave $100 \%$ sensitivity to Hp.

The online version of the original article can be found at https://oi.org/ $10.1007 / \mathrm{s} 00428-017-2205-0$

\footnotetext{
L. Mikhaleva

mikhalevalm@yandex.ru

Institute of Human Morphology, Moscow, Russia

2 City Clinical Hospital \#31, Moscow, Russia
} 